\title{
RACER: A data visualization strategy for exploring multiple genetic associations
}

Olivia L. Sabik ${ }^{1}$ and Charles R. Farber ${ }^{2}$

${ }^{1}$ Center for Public Health Genomics and Department of Biochemistry and Molecular Genetics, School of Medicine, University of Virginia, Charlottesville, VA 22908

${ }^{2}$ Center for Public Health Genomics and Departments of Public Health Science and Biochemistry and Molecular Genetics, School of Medicine, University of Virginia, Charlottesville, VA 22908

Summary: Genome-wide association studies (GWASs) have identified thousands of loci associated with risk of various diseases; however, the genes responsible for the majority of loci have not been identified. One means of uncovering potential causal genes is the identification of expression quantitative trait loci (eQTL) that colocalize with disease loci. Statistical methods have been developed to assess the likelihood that two associations (e.g. disease locus and eQTL) share a common causal variant, however, visualization of the two loci is often a crucial step in determining if a locus is pleiotropic. While the current convention is to plot two associations side-by-side, it is difficult to compare across two x-axes, even if they are identical. Thus, we have developed the Regional Association ComparER (RACER) package, which creates "mirror plots", in which the two associations are plotted on a shared x-axis. Mirror plots provide an effective tool for the visual exploration and presentation of the relationship between two genetic associations.

Availability and Implementation: RACER is provided under the GNU General Public License version 3 (GPL-3.0). Source code is available at https://github.com/oliviasabik/RACER.

Contact: ols5fg@virginia.edu

Supplementary information: Supplementary data are available online with the paper, see the Supplemental Data Manifest. 


\section{Introduction}

Genome-wide association studies (GWASs) have identified thousands of loci associated with disease risk; however, the genes responsible for the majority of these disease-associated loci remain largely unknown (Gallagher and Chen-Plotkin, 2018). A common approach to identify causal genes is to determine if disease-associated variants also influence molecular phenotypes, such as gene expression (Nicolae et al., 2010). This approach has become more widely implemented as expression quantitative trait loci (eQTL) across many tissues have become available from projects such as the Genotype-Tissue Expression Project (GTEx) (GTEx Consortium, 2013). Several statistical approaches that provide formal evidence of colocalization between two associations (e.g. a disease locus and eQTL) have been developed (Giambartolomei et al., 2018; Hormozdiari et al., 2016; Nica et al., 2010,https://github.com/Ksieber/piccolo); however, effective visualization is often an important component of colocalization analyses to ensure the presence of a single pleiotropic association. A common convention is to plot two associations separately using LocusZoom or LocusCompare and present them side by side, though it is often difficult to compare associations plotted on two different x-axes (Pruim et al., 2010, https://github.com/boxiangliu/locuscompare). To address this issue we built the Regional Association ComparER (RACER) package, which creates "mirror plots" for two individual associations. Mirror plots illustrate two associations, one inverted, on a shared $\mathrm{x}$-axis, allowing for the direct comparison of the associated variants for two phenotypes.

\section{RACER Features}

RACER was developed as a data visualization tool for the comparison of two sets of association data that share a common locus. With RACER, users can plot association data, minimally containing columns for chromosome, genomic coordinates, and p-values for an association. RACER contains a formatting function which can take any association data as input, and format it for compatibility with plotting functions. RACER also contains a function for annotating association data with population-specific linkage disequilibrium data from the 1000 genomes project using LD Link using reference SNP IDs or formatting existing linkage 
disequilibrium provided by the user for a specific study population (1000 Genomes Project Consortium et al., 2015; Machiela and Chanock, 2015). Once the association data has been formatted and annotated, RACER can produce three different types of plots: (1) a plot of a single association (Supplemental Figure 1), (2) a scatter plot of the p-values from two different association data sets (Supplemental Figure 2), or (3) a mirror plot for two associations (Figure 1). A vignette illustrating how to create the MARK3 eQTL/BMD association mirror plot described below can be found at https://oliviasabik.github.io/RACERweb/articles/IntroToRACER.html.

\section{RACER Application}

As a demonstration of the utility of RACER, we present a case using GTEx eQTL data to interrogate a locus on Chr. 14q32.32 associated with bone mineral density (BMD). The Chr. 14q32.32 locus spanned approximately $160 \mathrm{Kbp}$ and included three genes: MARK3, CKB and TRMT61A. We previously demonstrated that the expression of all three genes were influenced by significant eQTL $\left(p<1.0 \times 10^{-5}\right)$ in at least one GTEx tissue (Calabrese et al., 2017). In the original paper, we analyzed these relationships using GTEx release 6 and BMD GWAS data from a 2012 study (Estrada et al., 2012). To demonstrate the use of RACER, we performed a new analysis using GTEx release v7 and BMD GWAS data from a 2017 study (Supplemental Data 1, Supplemental Data 2)(GTEx Consortium et al., 2017; Kemp et al., 2017). First, we used the coloc $\mathrm{R}$ package to estimate the posterior probability (PPH4) that each pair of associations were due to single causal variants (Giambartolomei et al., 2018). Using coloc, we observed that both MARK3 and TRMT61A were likely to share a causal variant $(\mathrm{PPH} 4=95.9 \%$ and $\mathrm{PPH} 4=83.4 \%$, respectively). The likelihood that the CKB eQTL colocalizes with the Chr. 14q32.32 locus was low $(\mathrm{PPH} 4=21.3 \%)$

We used RACER to create mirror plots comparing the BMD association with each of the three eQTL. This visualization of the MARK3 and TRMT61A eQTL in direct comparison with the BMD association indicate that the MARK3 eQTL has an architecture more similar to the BMD association than the TRMT61A eQTL. The MARK3 eQTL is nearly identical to the BMD association; the same variants are the most significantly associated with both MARK3 expression and BMD, and the pattern of association is similar across SNPs of decreasing LD. 
bioRxiv preprint doi: https://doi.org/10.1101/495366; this version posted December 14,2018 . The copyright holder for this preprint (which was not certified by peer review) is the author/funder, who has granted bioRxiv a license to display the preprint in perpetuity. It is made available RACER: Data Visualization for Comparing Genetic Associations under aCC-BY-NC 4.0 International license.

While the TRMT61A eQTL and BMD association have a PPH4>75\%, which is considered sufficient evidence of a shared causal variant, it appears to be influenced by multiple associations in this region (Giambartolomei et al., 2018). The variants that are the most significantly associated with TRMT61A expression only exhibit low linkage disequilibrium with the SNPs that are the most significantly associated with BMD. However, the most significant BMD variants do see to be represented in the association, albeit at a lower level of significance. As observed in the coloc results, the CKB eQTL signal is dominated by an alternative signal, similar in architecture to the strongest signal in the TRMT61A eQTL. Using RACER, we confirmed the coloc results for $C K B$ and gained a more nuanced view of the TRMT61A and MARK3 results. Though this analysis does not exclude the involvement of TRMT61A or CKB, it does provide further evidence that MARK3 is responsible for the association.
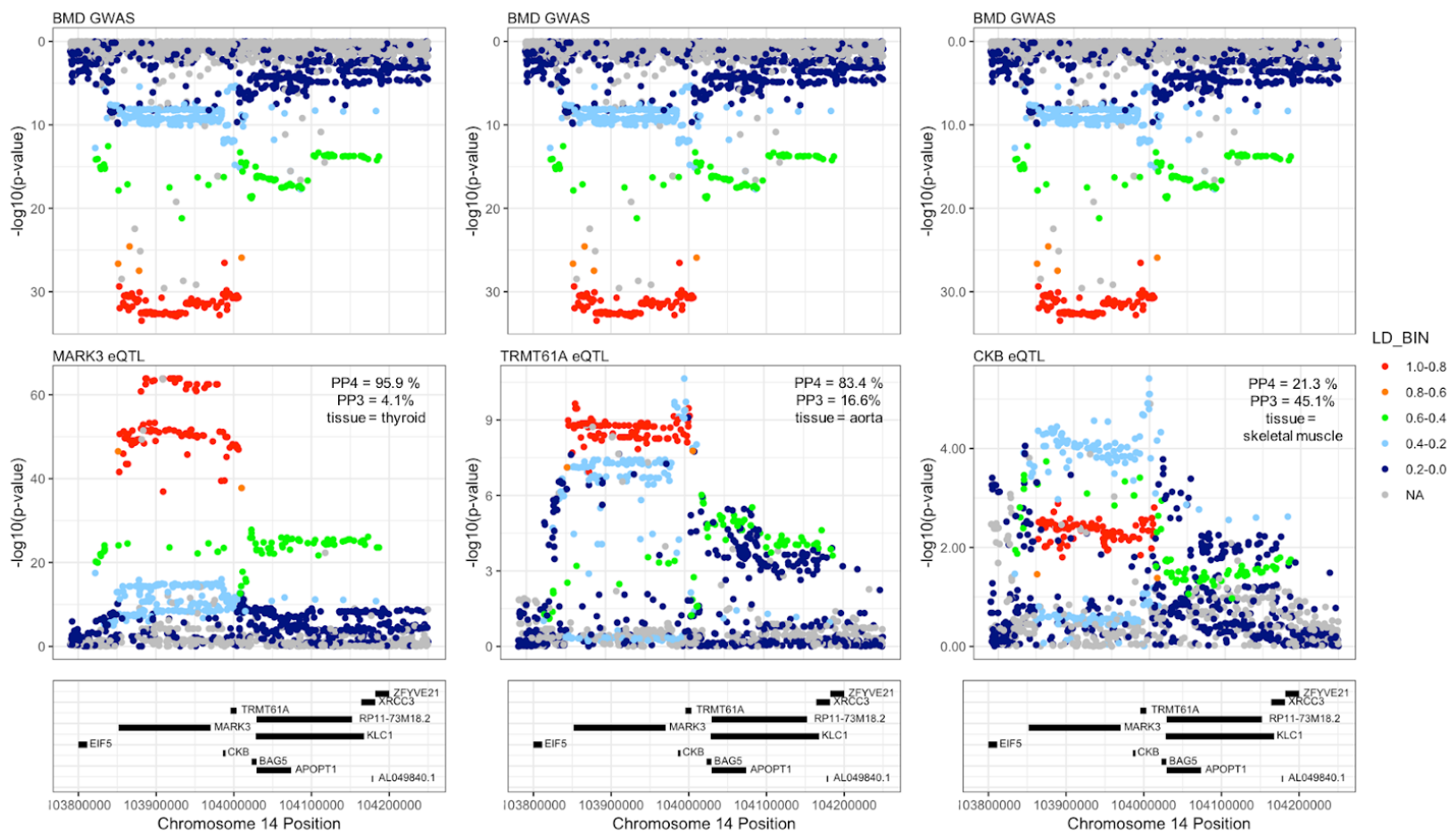

Figure 1. Mirror plots for MARK3, TRMT61A and CKB eQTL and a BMD GWAS locus. The mirror plots illustrate the similarity of the BMD association and MARK3 eQTL, the complexity of the TRMT61A eQTL, and the dominance of the secondary association in the CKB eQTL. 
While we demonstrated the comparison between a disease association and an eQTL, RACER can be used to visualize the comparison between any two associations at a common locus, including associations for different phenotypes which may arise from a pleiotropic variant, or comparable associations arising from studies carried out in populations of different ethnicities.

\section{Conclusion}

We have developed RACER, an R package to produce mirror plots, which allow for the direct comparison of two different associations within the same locus. Mirror plots provide an effective tool for the visual exploration and presentation of the relationship between two genomic associations.

\section{Acknowledgements}

We would like to thank Nathan Sheffield (University of Virginia) and John Lawson (University of Virginia) for their advice in the development of RACER, and Basel Al-Barghouthi (University of Virginia), Eric Taleghani (University of Virginia), and Catherine Robertson (University of Virginia), all of whom provided critical testing and input throughout the development of RACER.

\section{Funding}

OLS was supported by a Wagner Fellowship from the University of Virginia and the University of Virginia Cell and Molecular Biology Training Grant funded by the National Institute of General Medical Sciences (T32 GM8136-31A1). This work was also supported by the National Institute of Arthritis and Musculoskeletal and Skin Diseases of the National Institutes of Health [AR071657, AR064790, and AR068345 to CRF]. The Genotype-Tissue Expression (GTEx) Project was supported by the Common Fund of the Office of the Director of the National Institutes of Health, and by NCI, NHGRI, NHLBI, NIDA, NIMH, and NINDS. Conflicts of Interest: none declared.

\section{References}


1000 Genomes Project Consortium et al. (2015) A global reference for human genetic variation. Nature, 526, 68-74.

Calabrese, G.M. et al. (2017) Integrating GWAS and Co-expression Network Data Identifies Bone Mineral Density Genes SPTBN1 and MARK3 and an Osteoblast Functional Module. Cell Syst, 4, 46-59.e4.

Estrada, K. et al. (2012) Genome-wide meta-analysis identifies 56 bone mineral density loci and reveals 14 loci associated with risk of fracture. Nat. Genet., 44, 491-501.

Gallagher, M.D. and Chen-Plotkin, A.S. (2018) The Post-GWAS Era: From Association to Function. Am. J. Hum. Genet., 102, 717-730.

Giambartolomei, C. et al. (2018) A Bayesian framework for multiple trait colocalization from summary association statistics. Bioinformatics, 34, 2538-2545.

GTEx Consortium et al. (2017) Genetic effects on gene expression across human tissues. Nature, 550, 204-213.

GTEx Consortium (2013) The Genotype-Tissue Expression (GTEx) project. Nat. Genet., 45, 580-585.

Hormozdiari, F. et al. (2016) Colocalization of GWAS and eQTL Signals Detects Target Genes. Am. J. Hum. Genet., 99, 1245-1260.

Kemp, J.P. et al. (2017) Identification of 153 new loci associated with heel bone mineral density and functional involvement of GPC6 in osteoporosis. Nat. Genet., 49, 1468-1475.

Machiela, M.J. and Chanock,S.J. (2015) LDlink: a web-based application for exploring population-specific haplotype structure and linking correlated alleles of possible functional variants. Bioinformatics, 31, 3555-3557.

Nica, A.C. et al. (2010) Candidate causal regulatory effects by integration of expression QTLs with complex trait genetic associations. PLoS Genet., 6, e1000895.

Nicolae, D.L. et al. (2010) Trait-associated SNPs are more likely to be eQTLs: annotation to enhance discovery from GWAS. PLoS Genet., 6, e1000888.

Pruim, R.J. et al. (2010) LocusZoom: regional visualization of genome-wide association scan results. Bioinformatics, 26, 2336-2337. 